\title{
Precise tuning of the Curie temperature of (Ga,Mn)As-based magnetic semiconductors by hole compensation: Support for valence-band ferromagnetism
}

\author{
Shengqiang Zhou, ${ }^{1, *}$ Lin Li, ${ }^{1,2}$ Ye Yuan, ${ }^{1}$ A. W. Rushforth, ${ }^{3}$ Lin Chen,${ }^{4}$ Yutian Wang, ${ }^{1}$ \\ Jianhua Zhao, ${ }^{4}$ K. W. Edmonds, ${ }^{3}$ R. P. Campion, ${ }^{3}$ B. L. Gallagher ${ }^{3}$ C. Timm, ${ }^{5}$ and M. Helm ${ }^{1}$ \\ ${ }^{1}$ Helmholtz-Zentrum Dresden-Rossendorf, Institute of Ion Beam Physics and \\ Materials Research, Bautzner Landstrasse 40001328 Dresden, Germany \\ ${ }^{2}$ Department of Physics and Electronics, School of Science, Beijing University of Chemical Technology, Beijing 100029, China \\ ${ }^{3}$ School of Physics and Astronomy, University of Nottingham, Nottingham NG7 2RD, United Kingdom \\ ${ }^{4}$ Institute of Semiconductors, Chinese Academy of Science, Beijing 100080, China \\ ${ }^{5}$ Institute of Theoretical Physics, Technische Universität Dresden, 01062 Dresden, Germany
}

(Dated: June 15, 2016)

\begin{abstract}
For the prototype diluted ferromagnetic semiconductor (Ga,Mn)As, there is a fundamental concern about the electronic states near the Fermi level, i.e., whether the Fermi level resides in a well-separated impurity band derived from Mn doping (impurity-band model) or in the valence band that is already merged with the Mn-derived impurity band (valence-band model). We investigate this question by carefully shifting the Fermi level by means of carrier compensation. We use helium-ion implantation, a standard industry technology, to precisely compensate the hole doping of GaAs-based diluted ferromagnetic semiconductors while keeping the Mn concentration constant. We monitor the change of Curie temperature $\left(T_{C}\right)$ and conductivity. For a broad range of samples including $(\mathrm{Ga}, \mathrm{Mn})$ As and $(\mathrm{Ga}, \mathrm{Mn})(\mathrm{As}, \mathrm{P})$ with various $\mathrm{Mn}$ and $\mathrm{P}$ concentrations, we observe a smooth decrease of $T_{C}$ with carrier compensation over a wide temperature range while the conduction is changed from metallic to insulating. The existence of $T_{C}$ below $10 \mathrm{~K}$ is also confirmed in heavily compensated samples. Our experimental results are naturally explained within the valence-band picture.
\end{abstract}

\section{INTRODUCTION}

As one of three routes to spintronics [1], diluted ferromagnetic semiconductors (DFS) have been under extensive investigation for two decades [2-5]. Mn-doped $\mathrm{GaAs}$ [and its alloys such as $\mathrm{Ga}(\mathrm{As}, \mathrm{P})$ and $\mathrm{Ga}(\mathrm{As}, \mathrm{Sb})$ ] is regarded as a prototype. Spintronic devices [6-9], hybrid structures [10,11], and other spintronic phenomena [12-14] have been successfully demonstrated. One of the bottlenecks limiting its application is the low Curie temperature $T_{C}$, which still does not reach room temperature [15].

Different theoretical models suggest distinct strategies for increasing $T_{C}$. In its extreme limit, the impurityband picture assumes the existence of a separate impurity band formed by Mn-derived dangling-bond hybrid $(\mathrm{DBH})$ states $[4,16,17]$. In the presence of compensation, the Fermi energy falls into this impurity band. This picture is expected to be correct for weakly doped samples. On the other hand, the extreme valence-band picture proposes that the would-be impurity band is so strongly broadened that it merges with the valence band and does not leave a distinct maximum in the density of states (DOS). The only effect of Mn doping, besides moving the Fermi energy into the merged band, is to create a tail of localized states at the band edge $[2,4]$. If compensation is not too strong, the states at the Fermi energy are extended and have valence-band character with some ad-

* Electronic address: S.Zhou@hzdr.de mixture of DBHs. There is a continuum of cases connecting these two extremes and some of the controversy [18$26]$ in the field seems to result from overinterpreting the two pictures. In this work, we use the term "impurityband picture" to refer to a situation where the impurity band may have merged with the valence band but is still visible by a clear maximum in the DOS. This means that in uncompensated systems, the Fermi energy falls into a minimum of the DOS, where the electronic states show an enhanced tendency towards localization. The low DOS and the localization generically lead to reduced conductivity and magnetic coupling. Hence, within the impurity-band model $T_{C}$ is expected to decrease as compensation goes to zero, whereas partial compensation of holes promotes ferromagnetic order and $T_{C}$ reaches its maximum when the impurity band is roughly half filled $[25,27]$. Within the valence-band picture, one instead expects the DOS and $T_{C}$ to increase monotonically with hole concentration. In the extreme limit, Dietl et al. [28] and Jungwirth et al. [20] indeed predict, within meanfield theory, that $T_{C}$ increases monotonically with substitutional Mn concentration and with hole concentration.

Even after more than a decade, the question of where the holes are residing is still being debated [18-26]. One reason for this is that some experimental probes might be more susceptible to DBH-derived properties (for example, local probes at Mn sites) while others see more of the valence-band physics (e.g., transport). Another reason is that the preparation of heavily Mn-doped GaAs is a very delicate procedure, which may result in large variations of sample composition and quality [29, 30]. Furthermore, $\mathrm{Mn}$ in GaAs acts as an acceptor, resulting in the dif- 
ficulty to independently control the local-moment and hole concentrations. On the other hand, interstitial Mn ions $\left(\mathrm{Mn}_{\mathrm{I}}\right)$ are double donors, which, intentionally or unintentionally, compensate the hole doping. Independent precise control of both moment density and hole concentration is required to resolve the "band battle" [26].

Four different approaches have been applied to adjust the hole concentration while keeping the Mn concentration constant. Myers et al. [31] utilized the As-antisite effect to compensate holes in the low-doping regime of 1-2\% Mn. During growth, they intentionally did not rotate the substrate to obtain a variation of the As to Ga flux ratio, and thereby of the hole concentration, across a single wafer. The second approach is to compensate holes in $(\mathrm{Ga}, \mathrm{Mn})$ As by exposing the samples to a hydrogen plasma [32], followed by post-annealing [3335]. In this way, the magnetic and electronic properties of $(\mathrm{Ga}, \mathrm{Mn}) \mathrm{As}$ can be changed qualitatively, though without fine control. On the other hand, the third approach, electrical gating of a $(\mathrm{Ga}, \mathrm{Mn})$ As metal-insulatorsemiconductor structure, is expected to allow for a fine control over the hole concentration [36], but the required large gate voltage limits this method to samples with relatively low $T_{C}$ [23]. Finally, it is possible to tune the hole concentration in $(\mathrm{Ga}, \mathrm{Mn}) \mathrm{As}$ by ion irradiation [37-39], which is a standard industry method and allows for a fine tunability and reproducibility. This method is widely used for conventional III-V semiconductors to render a conducting layer highly resistive through the creation of carrier-trapping centers [40, 41]. It has been demonstrated to be applicable to (Ga,Mn)As [37-39] and (Ga,Mn)P [42].

In this contribution, we aim to shed light on the debate over the impurity vs. valence-band picture for (Ga,Mn)As-based DFS with high Mn concentration. Our approach is to examine the magnetic and transport properties while shifting the Fermi level to higher energies by compensating the free carriers. We demonstrate the possibility of fine-tuning the magnetism of highly conducting (Ga,Mn)As films by ion irradiation. With increasing the displacement per atom (DPA), the (Ga,Mn)As films become gradually more resistive. For a broad range of $(\mathrm{Ga}, \mathrm{Mn}) \mathrm{As}$ and also $(\mathrm{Ga}, \mathrm{Mn})(\mathrm{As}, \mathrm{P})$ samples, we find universally that $T_{C}$ distinctly and monotonically decreases with increasing DPA, which we interpret in terms of a decreasing free-hole concentration. Our samples are clearly distinct from those studied in Ref. 38, where the magnetization curves become more concave and nonBrillouin-function-like with increasing DPA, while $T_{C}$ changes relatively little. Even in the strongly insulating regime, we observe values of $T_{C}$ in the range $5_{-}^{-}$ $10 \mathrm{~K}$, unlike in Ref. 43 , where $T_{C}$ suddenly drops to $0 \mathrm{~K}$ from around 10-20 K. Taken together with the other observables, e.g., the coercive field and the shape of the magnetization curves, our observations are naturally explained in terms of the valence-band picture but not of the impurity-band picture. This provides support for the former for ferromagnetic (Ga,Mn)As with high Mn con- centrations.

\section{EXPERIMENT}

\section{A. Sample preparation}

(Ga,Mn)As films were prepared at the University of Nottingham (UoN), and the Institute of Semiconductors (IOS), Chinese Academy of Sciences. All samples were grown by low-temperature molecular beam epitaxy (MBE) on semi-insulating GaAs(001). The details of the growth of the specific samples is described in the following.

Samples Mn6ann and Mn6ag were grown on lowtemperature GaAs buffer layers with a nominal Mn concentration of $x=0.06$ and with a thickness of $25 \mathrm{~nm}$ using a Veeco Mod Gen III MBE system at UoN [44]. The Mn concentration was determined from the Mn/Ga flux ratio. The material quality was found to strongly depend on the growth temperature and on the As/Ga flux ratio. The growth temperature of $230{ }^{\circ} \mathrm{C}$ is chosen to be the highest possible while maintaining $2 \mathrm{D}$ growth, as monitored by reflection high-energy electron diffraction. The films were grown under relatively low As flux to minimize the concentration of As antisites $\left(\mathrm{As}_{\mathrm{Ga}}\right)$. The use of $\mathrm{As}_{2}$ as a source instead of $\mathrm{As}_{4}$ also results in lower $\mathrm{As}_{\mathrm{Ga}}$ concentrations [45]. Sample Mn6ann was annealed in air at $190^{\circ} \mathrm{C}$ for 48 hours to remove compensating $\mathrm{Mn}_{\mathrm{I}}$. Sample Mn6ag was kept in the as-grown state.

The heavily Mn-doped (Ga,Mn)As film, sample Mn10ann, was grown at $200^{\circ} \mathrm{C}$ with a nominal Mn concentration of $x=0.10$ and a thickness of $20 \mathrm{~nm}$ at IOS [46]. During growth, the As/Ga beam equivalent pressure ratio was set to 8 . After growth, the sample was annealed at $200^{\circ} \mathrm{C}$ for 2 hours in air.

P-alloyed $(\mathrm{Ga}, \mathrm{Mn})(\mathrm{As}, \mathrm{P})$ samples were grown at UoN. A $50 \mathrm{~nm}$ low-temperature $\mathrm{GaAs}_{1-y} \mathrm{P}_{y}$ buffer layer was grown right before a $25 \mathrm{~nm} \mathrm{Ga} \mathrm{Ga}_{0.9} \mathrm{Mn}_{0.06} \mathrm{As}_{1-y} \mathrm{P}_{y}$ layer, with nominal concentrations $y=0.06$ and $y=0.09$ for samples Mn6P6ann and Mn6P9ann, respectively. The buffer and Mn-containing layers were all grown at a low temperature of $230^{\circ} \mathrm{C}$. Both were annealed in air at $190^{\circ} \mathrm{C}$ for 48 hours.

The sample definitions and the most important parameters are listed in Tab. I.

\section{B. Ion irradiation}

All (Ga,Mn)As wafers were cut into pieces of dimensions of about $5 \times 5 \mathrm{~mm}^{2}$ for ion irradiation. The He-ion energy was chosen as $4 \mathrm{keV}$ (a few as $650 \mathrm{keV}$ ). The details of the irradiation conditions are given in table II in App. A. During ion irradiation, the samples were tilted by $7^{\circ}$ to avoid the channeling effect. The samples were pasted onto Si wafers, which were kept at around room temperature without cooling or heating. The resulting 
TABLE I. Sample definitions and related parameters. The ending in Sample ID: "ann" means that the sample was annealed at low temperatures for long time, while "ag" means that the samples was as grown.

\begin{tabular}{ccccc}
\hline \hline Sample ID & Stoichiometry & Thickness & Nominal Mn concentration & Initial $T_{C}$ \\
\hline Mn6ann & $(\mathrm{Ga}, \mathrm{Mn}) \mathrm{As}$ & $25 \mathrm{~nm}$ & 0.06 & 0.06 \\
Mn6ag & $(\mathrm{Ga}, \mathrm{Mn}) \mathrm{As}$ & $25 \mathrm{~nm}$ & 0.10 & $60 \mathrm{~K}$ \\
Mn10ann & $(\mathrm{Ga}, \mathrm{Mn}) \mathrm{As}$ & $20 \mathrm{~nm}$ & 0.06 & $150 \mathrm{~K}$ \\
Mn6P6ann & $(\mathrm{Ga}, \mathrm{Mn}) \mathrm{As}_{0.94} \mathrm{P}_{0.06}$ & $25 \mathrm{~nm}$ & 0.06 & $125 \mathrm{~K}$ \\
Mn6P9ann & $(\mathrm{Ga}, \mathrm{Mn}) \mathrm{As}_{0.91} \mathrm{P}_{0.09}$ & $7 \mathrm{~nm}$ & $\mathrm{~K}$ \\
\hline \hline
\end{tabular}

defects were found to be distributed roughly uniformly through the whole (Ga,Mn)As layer by simulation using the SRIM (Stopping and Range of Ions in Matter) code [47]. In the following, we refer to the displacement per atom (DPA), which is the number of times that an atom in the target is displaced during irradiation. The DPA is a function of projectile type (ion specimens, neutron, or light energetic particles), energy, fluence, and ion charge as well as material properties. In the literature, the calculated DPA is often used as a measure of the irradiation effect in materials. As an example, for GaAs, 14 He ions of $4 \mathrm{keV}$ will produce a similar DPA as one $\mathrm{Ne}$ ion of $20 \mathrm{keV}$ at the same depth. The DPA is a better representation of the effect of irradiation on materials properties than ion fluence (dosage). It allows for a comparison between our results and data reported in the literature, in which often different ion specimens and energies are used.

By Rutherford backscattering spectroscopy in channeling geometry, which is sensitive to point defects such as Mn interstitials [48-50], we do not observe measurable increase of Mn interstitials even for the largest DPA applied in our work (see App. B). In the work of Winkler et al. [42], the structural integrity of (Ga,Mn)P after Ar-ion irradiation was confirmed by several techniques. Using ion channeling, the sheet concentration of $\mathrm{Mn}_{\mathrm{Ga}}$ in their samples was found to remain constant. High-resolution transmission electron microscopy and atomic force microscopy similarly showed no qualitative changes with ion irradiation. Therefore, we can conclude that the effect of He-ion irradiation is mainly to introduce deep traps and thereby compensate the holes. The reduction of hole concentration upon ion irradiation linearly depends on the ion fluence, i.e., the DPA [37, 42].

\section{Measurements}

Magnetic properties were measured with a superconducting quantum interference device (Quantum Design, SQUID-MPMS or SQUID-VSM) magnetometer. To determine $T_{C}$, we have measured the temperaturedependent magnetization at a small field of 10 or $20 \mathrm{Oe}$ after cooling down in field. Magnetotransport properties were measured with a magnetic field applied perpendicularly to the film plane in van der Pauw geometry using a commercial Lakeshore Hall System. Fields up to $80 \mathrm{kOe}$ were applied over a wide temperature range from $2.5 \mathrm{~K}$ to $300 \mathrm{~K}$.

\section{RESULTS AND DISCUSSION}

Figure 1 shows representatively the temperaturedependent magnetization and the magnetization vs. field at $5 \mathrm{~K}$ for three samples listed in Tab. I before and after irradiation at various ion fluences. All measurements were carried out with the field along the magnetic easy axis, which is GaAs[1ํㅣ, except for sample Mn6P9ann, where it is GaAs[001] (out-of-plane). Figure 2 shows the Curie temperature $T_{C}$ and the coercive field $H_{C}$ as functions of DPA for all samples. Several features can be observed, which will be discussed in detail below:

- $T_{C}$ monotonically decreases with increasing compensation of holes by ion irradiation over a broad range of DPA.

- The shape of magnetization vs. temperature remains convex from above.

- There is no significant difference between the behavior of samples Mn6ann and Mn6P6ann even though the sample Mn6P6ann contains $6 \% \mathrm{P}$.

\section{A. $T_{C}$ vs. compensation}

Let us first discuss the plausible picture regarding the hole compensation in our samples. We define $p$ as the absolute hole concentration and $f$ as the ratio between the hole and Mn concentrations, $f=0,0.5,1$ meaning full, half, and zero compensation, respectively. The samples Mn6ann, Mn10ann, and Mn6P6ann show rather high $T_{C}$ and are only weakly compensated by $\mathrm{Mn}_{\mathrm{I}}$ or other defects before irradiation. For samples Mn6ann and Mn6P6ann, $p$ has been carefully measured through the Hall effect, showing that $f$ is definitely above 0.5 , see Ref. 51 . Moreover, the saturation magnetization for sample Mn6ann (non-irradiated) is around $3.32 \mu_{B} / \mathrm{Mn}$. The value is nearly the same as that for state-of-the-art high-quality (Ga,Mn)As [52]. According to Ref. 52, the hole concentration is nearly the same as the effective Mn concentra- 

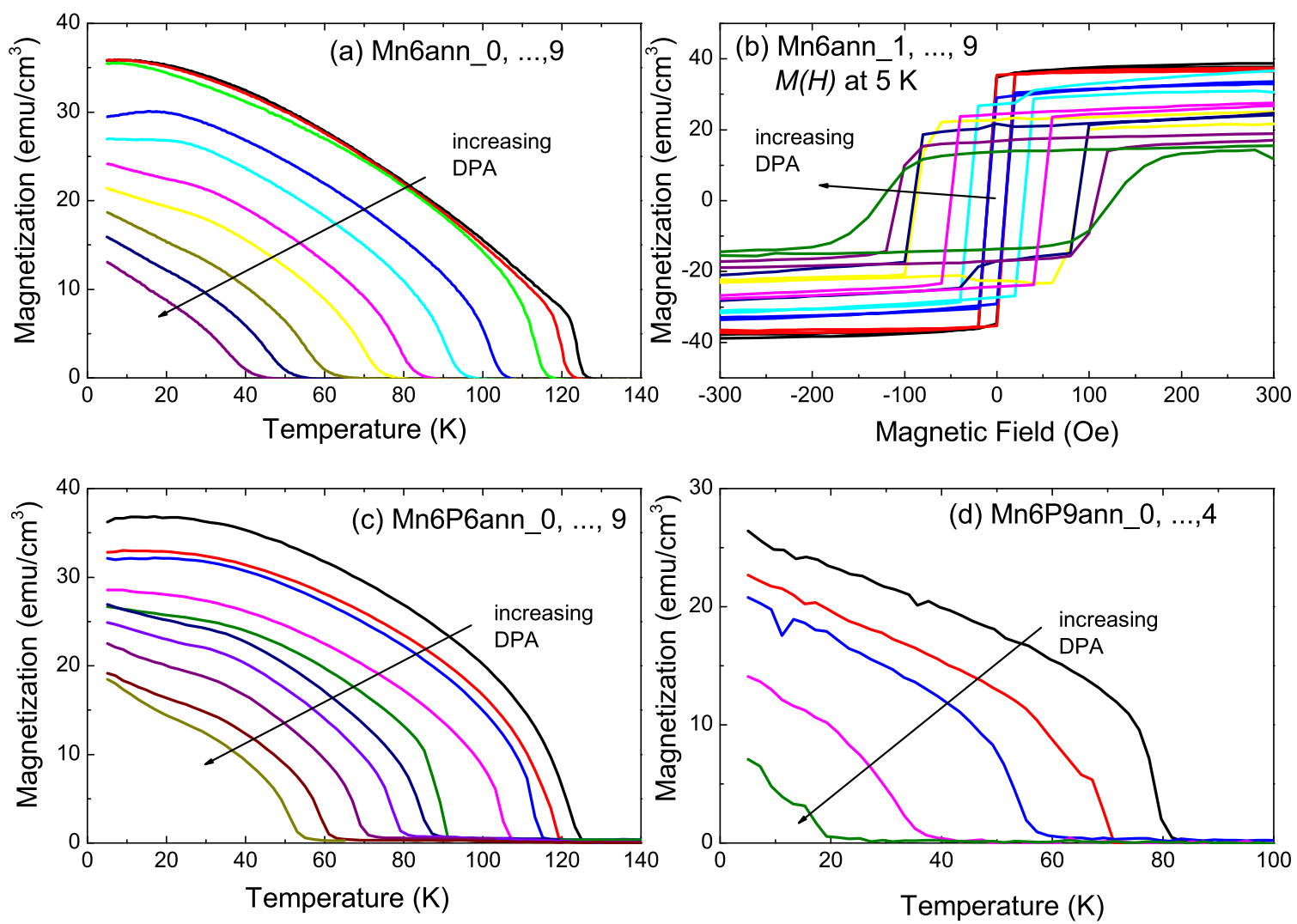

FIG. 1. Magnetic properties after introducing hole compensation by ion irradiation. The ion fluence was increased in linear steps. The hole concentration is expected to decrease linearly depending on DPA [37, 42]. Panels (a), (c) and (d) show the temperature dependent magnetization for different samples, while panel (b) shows the magnetic hysteresis for sample Mn6ann for various ion fluences. The temperature-dependent magnetization is measured at a small field of 20 Oe after cooling in field. One observes an increase in the coercive field $H_{C}$ when $T_{C}$ and the remanent magnetization decrease. The arrows indicate the increase of DPA from 0 to $2.88 \times 10^{-3}$. The corresponding DPA values are shown in Fig. 2 . In each panel, the black line is the result for the non-irradiated sample.

tion, $f \approx 1$. The impurity-band picture naturally predicts a strong suppression of carrier-mediated ferromagnetism close to $f=1$, since then the Fermi energy would fall into a band gap, a mobility gap, or at least an energy range of reduced DOS between the valence band and the purported impurity band [3]. Within the impurityband framework, we should thus see an increase in $T_{C}$ as the compensation approaches $f \approx 0.5$. Within the mean-field virtual crystal approximation [53], $T_{C}$ is proportional to the $\mathrm{Mn}_{\mathrm{Ga}}$ concentration, to $p^{1 / 3}$, and to the effective mass $m^{*}$. If the hole-hole exchange interactions are included, $T_{C}$ can be slightly enhanced by $10-20 \%$. Therefore, in the valence-band picture, $T_{C}$ is expected to decrease with decreasing hole concentration, regardless of the initial compensation. This is indeed what we find: $T_{C}$ universally decreases with increasing DPA for all sam- ples. In App. ??, we calculate the hole concentration $p$ for sample Mn6ann by following the approach described in Ref. 37. Indeed, the dependence of $T_{C}$ on $p$ is insensitive to the power $1 / 3$ or 1 when the hole concentration is large. Nevertheless, $T_{C}$ reveals a linear dependence on $p^{1 / 3}$, as expected in the framework of the valence-band picture.

\section{B. Phosphorus co-alloying}

Phosphorus co-alloying in ( $\mathrm{Ga}, \mathrm{Mn}) \mathrm{As}$ has been employed to tune the magnetic anisotropy [54-57], the Fermi energy [58], and the spin stiffness [59]. It is instructive to consider samples Mn6P6ann and Mn6P9ann, which are co-alloyed with $6 \%$ and $9 \%$ phosphorus, respectively. 

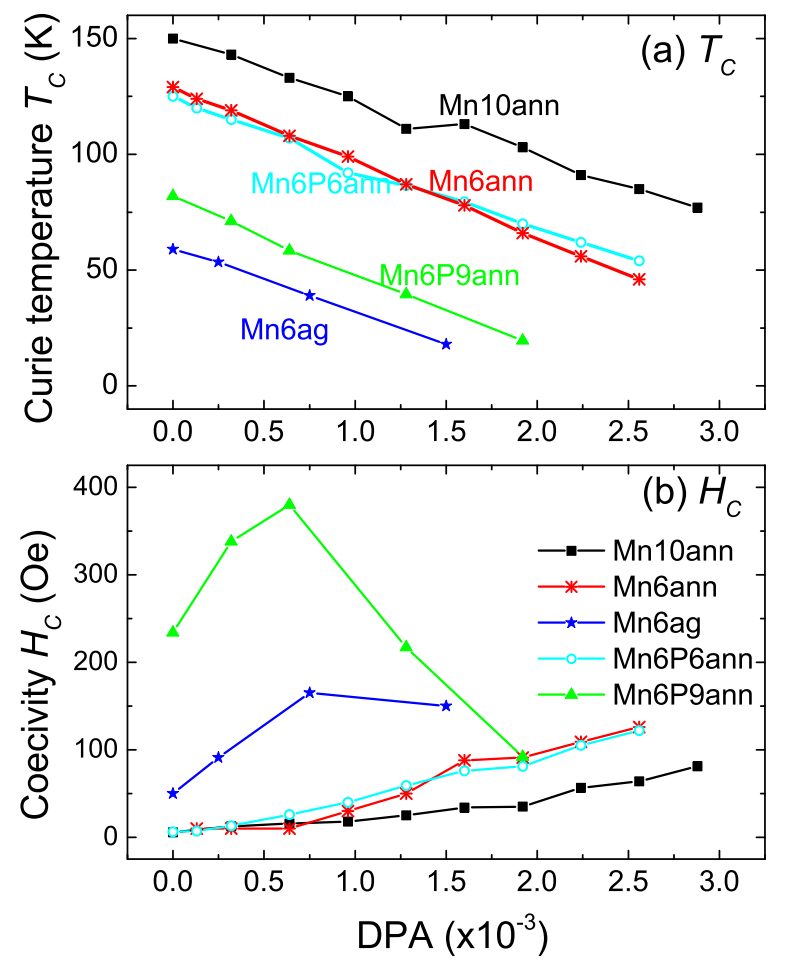

FIG. 2. (a) Curie temperature $T_{C}$ vs. DPA (ion fluence). All samples show a monotonic decrease in $T_{C}$. (b) Coercive field $H_{C}$ vs. DPA (ion fluence). For the samples with higher $T_{C}$, i.e., less compensation (samples Mn6ann, Mn10ann, Mn6P6ann), $H_{C}$ shows a monotonic increase with decreasing hole concentration.

In the nonirradiated samples, we do not see significant differences in magnetization and $T_{C}$ between samples Mn6P6ann and Mn6ann, which contains the same nominal concentration of $\mathrm{Mn}$ but no $\mathrm{P}$. This strongly differs from the work by Stone et al. [60], who observed a significant reduction of $T_{C}$ upon $\mathrm{P}$ co-alloying. They also found that ferromagnetic $\mathrm{Ga}_{0.954} \mathrm{Mn}_{0.046} \mathrm{As}$ films are changed from metallic to insulating by around $2.4 \% \mathrm{P}$ coalloying, which is attributed to the fact that holes located within an impurity band are scattered by alloy disorder in the anion sublattice [60]. However, our P-alloyed sample Mn6P6ann is metallic even though the $\mathrm{P}$ concentration is $6 \%$. Moreover, comparing samples Mn6ann with Mn6P6ann, our results reveal similar magnetization, $T_{C}$, and their dependence on the hole compensation. On the other hand, sample Mn6P9ann exhibits a remarkable reduction in $T_{C}$ compared to samples Mn6ann and Mn6P6ann, likely due to its larger concentration of $\mathrm{P}$. In the work by Cubukcu et al. [58], the energy level of Mn moves deeper into the gap and the transport character changes from metallic to insulating with increasing
$\mathrm{P}$ concentration. The Curie temperature drops sharply from $130 \mathrm{~K}$ to $45 \mathrm{~K}$ when the $\mathrm{P}$ concentration is increased from 0 to around $19 \%$. The extreme representatives are $(\mathrm{Ga}, \mathrm{Mn}) \mathrm{P}$ and $(\mathrm{In}, \mathrm{Mn}) \mathrm{P}$, in which the Mn energy levels are located $400 \mathrm{meV}$ and $220 \mathrm{meV}$ above the valence-band edge, respectively, compared to $110 \mathrm{meV}$ for $(\mathrm{Ga}, \mathrm{Mn}) \mathrm{As}$. Both materials exhibit hopping conduction [61, 62].

A theoretical work by R. Bouzerar et al. [63] explains the large reduction of $T_{C}$ for $\mathrm{P}$ co-alloyed ( $\left.\mathrm{Ga}, \mathrm{Mn}\right) \mathrm{As}$ reported in Ref. 60. They employ the local spin-density approximation to obtain Mn-Mn exchange interactions. These interactions are used in a Heisenberg model with random Mn positions, which is treated by the selfconsistent random-phase approximation [64] and complementary Monte Carlo simulations. Compensating defects- $\mathrm{P}$ antisites as well as Mn and P interstitials - lead to the reduction of the hole concentration and of $T_{C}$ compared to ( $\mathrm{Ga}, \mathrm{Mn})$ As not containing P. If compensating defects can be avoided, the change in $T_{C}$ is expected to be negligible for low $\mathrm{P}$ concentration $[58,65]$, which is indeed in good agreement with our MBE-grown samples.

\section{Magnetization and coercivity vs. compensation}

The reduction of the remanent magnetization $M$ at low temperatures, shown in Fig. 1, is still puzzling. It cannot be explained by a loss of substitutional Mn ions. Note that we applied He-ion irradiation with very low fluences $\left(10^{12}-10^{13} \mathrm{~cm}^{-2}\right)$. The maximal DPA is only $0.2-$ $0.3 \%$ according to SRIM simulations [47]. Most of the substitutional Mn ions are expected to remain at their original sites, as we do not see any change in the structural characterization, see App. B. Therefore, within the valence-band framework the magnetization at zero temperature for the compensated samples is expected to stay the same as for the initial sample. Yet we see a significant reduction in both the remanent and the low-temperature saturation magnetization. At the largest fluence applied to sample Mn6ann, see Fig. 1(b), the magnetization is reduced by more than $50 \%$. The reduction of magnetization has been also observed in hydrogenated $(\mathrm{Ga}, \mathrm{Mn}) \mathrm{As}$ with reduced hole concentration [34] and can be reversed by annealing [35]. This phenomenon can be understood by considering the inhomogeneity of $(\mathrm{Ga}, \mathrm{Mn}) \mathrm{As}$ as suggested by Dietl [66] and by Sawicki et al. [23]. Carriermediated ferromagnetism is strongly influenced by the vicinity of the insulator-to-metal transition (IMT). Particularly, the spatial fluctuations in the local DOS can destroy the spatial homogeneity of the ferromagnetic order, resulting in a nanoscale phase separation. We consider a similar scenario: when defects are introduced, regions with an initially small hole concentration are depleted first and become paramagnetic or superparamagnetic. Therefore, a nanoscale phase separation regarding both the effective Mn concentration and the hole concentration can lead to the gradual appearance of superparamagnetic and paramagnetic regions, consequently reduc- 
ing the total magnetization. Indeed, we have observed a superparamagnetic component in sample Mn6ag with the lowest initial $T_{C}$ after irradiation up to large $\mathrm{He}$ fluences by measuring the zero-field-cooled/field-cooled temperature-dependent magnetization (see App. C).

Another reason for a decrease in the magnetization is the occurrence of antiferromagnetically coupled nearestneighbor $\mathrm{Mn}_{\mathrm{Ga}}$ pairs when the sample is heavily compensated $[53,67]$. The antiferromagnetically coupled pairs are invisible in the magnetization even at larger fields since the short-range antiferromagnetic interaction is large compared to the Zeeman energy.

The reduction of the remanent and apparent saturation magnetization with hole compensation naturally leads to an increase in the coercive field $H_{C}$. A consistent increase in $H_{C}$ with DPA for samples Mn6ann, Mn10ann, and Mn6P6ann is indeed seen in Fig. 2. The increase in $H_{C}$ agrees with predictions obtained from a valence-band description [68], lending additional support to this picture. Note that $H_{C}$ starts to decrease for samples Mn6P9ann and Mn6ag when the samples are subjected to much larger DPA. It is due to the fact that $T_{C}$ for those samples are already very low and only slightly above $5 \mathrm{~K}$, the measurement temperature for $H_{C}$ shown in Fig. 2. On the other hand, the magnetic anisotropy can also change when the compensation is high [34, 35, 39]. In hydrogenation-compensated $(\mathrm{Ga}, \mathrm{Mn}) \mathrm{As}$, the coercivity for magnetic field in the in-plane direction also increases [35].

\section{Resistivity vs. compensation}

Corresponding effects are observed in the temperaturedependent resistivity, as shown in Fig. 3. We present data for three samples: Mn6ann, Mn6P6ann and Mn6P9ann. Mn6P9ann is closer to the insulating regime due to the high concentration of $\mathrm{P}$. With increasing compensation, the resistivity for samples Mn6ann and Mn6P6ann continuously increases and develops an upturn at low temperatures, but both samples still show a broadened cusp around the Curie temperature. For sample Mn6P9ann, the cusp is present for low ion fluence but is overwhelmed by a huge low-temperature increase of the resistivity for moderate fluences. Note the very different relative change of the resistivity value at low temperatures: for samples Mn6ann and Mn6P6ann it changes by less than two orders of magnitude, whereas for sample Mn6P9ann it changes by more than five orders of magnitude. We have also measured the Hall resistance at $300 \mathrm{~K}$ (above $T_{C}$ ) for our samples, examples are shown in App. D. After irradiation, all samples still show p-type conductivity. The Hall coefficient at $300 \mathrm{~K}$ increases gradually with increasing DPA, see Fig. ?? in App. D. The results indicate a decrease of the hole concentration, but do not give an accurate measurement due to the paramagnetic component in the Hall effect [51]. However, Mayer et al. [37] have used electrochemical capacitance voltage profiling to

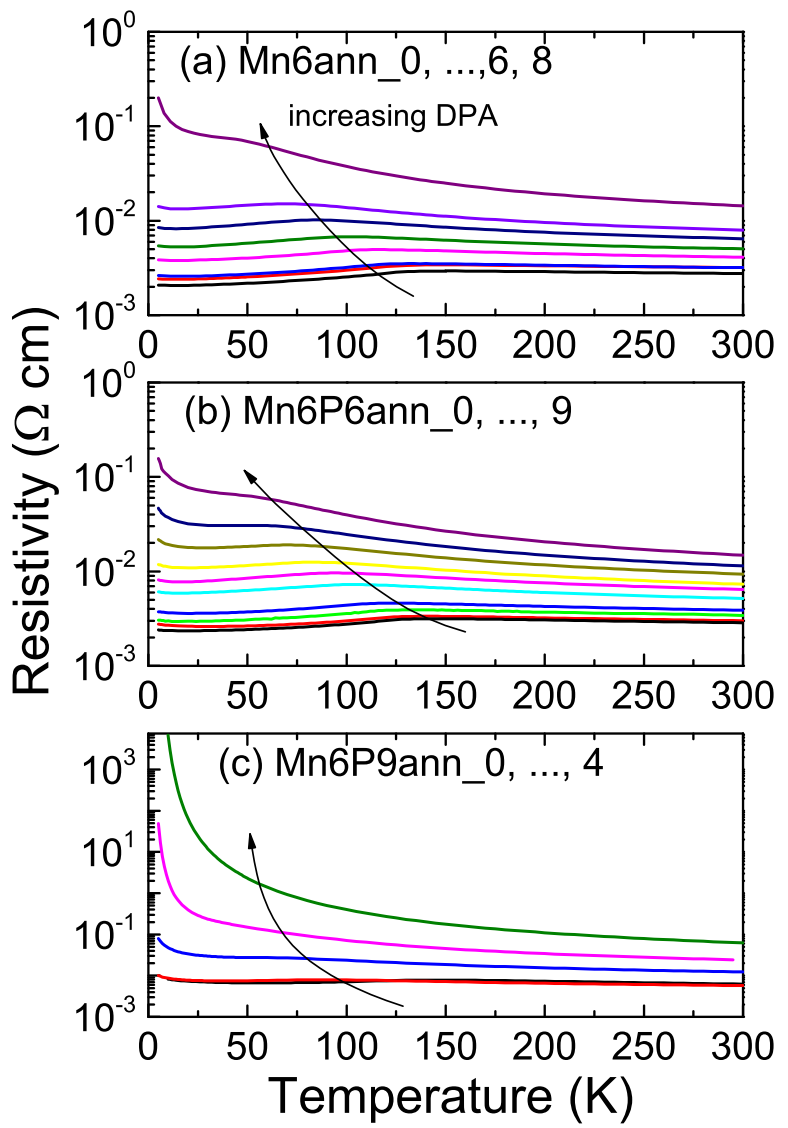

FIG. 3. Temperature-dependent resistivity in zero field for three samples upon ion irradiation. For samples Mn6ann (a) and Mn6P6ann (b), the resistivity continuously increases with decreasing hole concentration but still shows a broadened cusp around $T_{C}$, while sample Mn6P9ann (c) is closer to the insulating regime. The arrows indicate the increase of the DPA from 0 to $2.88 \times 10^{-3}$. The corresponding DPA values are shown in Fig. 2.

measure the hole concentration and have confirmed a linear correlation between hole concentration and DPA. We conclude that the moderate increase of resistivity in sample Mn6P6ann is mainly due to the reduction of the free hole concentration, while only at low temperatures an upturn due to carrier localization develops. On the other hand, the large resistivity increase for sample Mn6P9ann with a low initial $T_{C}$ is dominated by localization.

\section{E. Onset of ferromagnetism}

Within the Zener $p$ - $d$ exchange scenario, the Curie temperature should continuously decrease with decreasing hole concentration even into the insulating regime, since the electronic properties at the length scale of the typical Mn-Mn separation change continuously with hole concentration through the IMT $[3,23,28]$. Actually, Curie temperatures as low as $0.75 \mathrm{~K}$ to $2.4 \mathrm{~K}$ for p-doped 

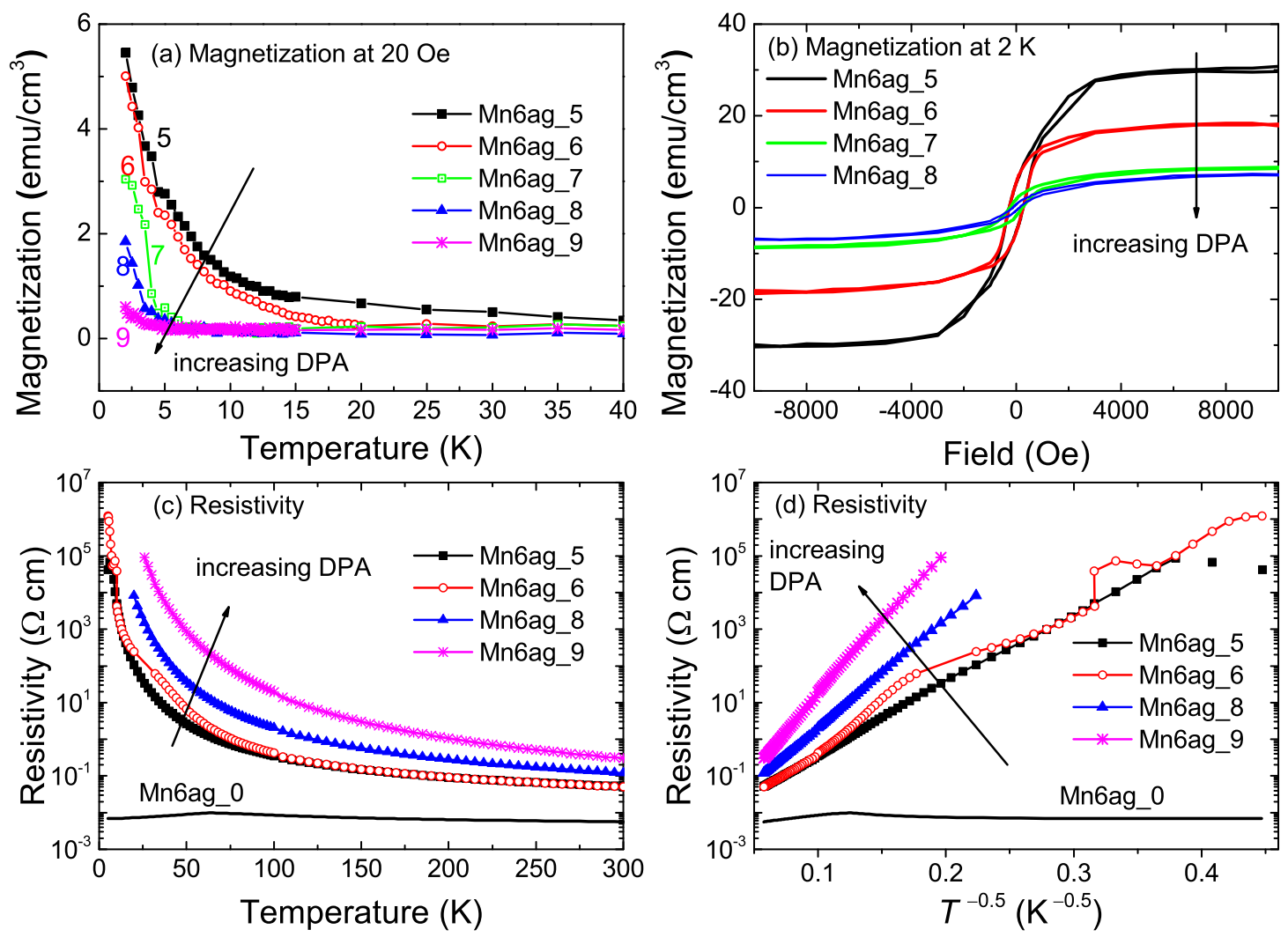

FIG. 4. Change of magnetic and transport properties of sample Mn6ag with compensation, close to the destruction of ferromagnetism. (a) Temperature dependent magnetization in a field of 20 Oe, (b) Magnetization vs. field at $2 \mathrm{~K}$. (c) and (d) temperature-dependent longitudinal resistance in zero field. A virgin, nonirradiated sample (Mn6ag_0) is shown for comparison. Mn6ag_5, .., Mn6ag_9 label the samples with different DPA.

(Zn,Mn)Te [69] and from $5 \mathrm{~K}$ to $8 \mathrm{~K}$ for (In,Mn)Sb [70] were measured.

Within the impurity-band picture [43], the interaction between Mn ions is double-exchange via the hopping of holes between two Mn ions. Therefore, the free-hole concentration $p$ has only a weak effect on $T_{C}$. Instead, the hopping energy should be a good predictor for ferromagnetism [43]. However, while the experimental data [43] show that ferromagnetic order only occurs within a certain range of hopping energies, the correlation between $T_{C}$ and the hopping energy is weak. The theory framework described in Ref. [43] predicts a discontinuous disappearance of the ferromagnetic state. Indeed, the experimental $T_{C}$ for $(\mathrm{Ga}, \mathrm{Mn}) \mathrm{As}$ is reported to jump from $10-15 \mathrm{~K}$ to zero.

The Anderson-Mott IMT occurs at a critical carrier concentration $p_{c}$. Empirically, the magnitude of $p_{c}$ is in the range $p_{c}^{1 / 3} a_{B}=0.26 \pm 0.05[1]$, where $a_{B}$ is the effective Bohr radius. $a_{B}$ can be evaluated from the impurity binding energy $E_{I}$ by $a_{B}=\hbar /\left(2 m^{*} E_{I}\right)^{1 / 2}$ or $a_{B}=e^{2} / 8 \pi \epsilon_{0} \epsilon_{r} E_{I}[1,71]$. For details, see Ref. 1. For
GaAs, the Mn single-impurity binding energy is $110 \mathrm{meV}$, but for InSb it is as low as several meV. Therefore, one can expect a much larger $p_{c}$ for $(\mathrm{Ga}, \mathrm{Mn})$ As than for $(\mathrm{In}, \mathrm{Mn}) \mathrm{Sb}$. The double role of $\mathrm{Mn}$ ions in GaAs as both local spins and acceptors usually prevents fine tuning of the carrier concentration near the IMT. This may explain the absence of the observation of a continuous decay of $T_{C}$ to $0 \mathrm{~K}$ for $(\mathrm{Ga}, \mathrm{Mn}) \mathrm{As}$. We expect that a (Ga,Mn)As sample with high Mn concentration but with very low hole concentration shows a $T_{C}$ close to zero. We choose sample Mn6ag, which has a relatively low initial $T_{C}$. Five pieces of sample Mn6ag were irradiated with He ions. The ion fluence was increased in very small step starting from a DPA of $1.5 \times 10^{-3}$ shown in Fig. $2(\mathrm{a})$, which leads to the hole concentration decreasing in small steps. Figure 4 shows the magnetic and transport properties of this set of samples. For all of them, the temperature-dependent remanent magnetization is concave from above, distinct from Fig. 1. This is typical for DFS with Fermi energy deep in the localized states in the disorder-induced band tail [72]. For these concave magnetization curves, it is difficult to extract pre- 
cise values for $T_{C}$. Nevertheless, $T_{C}$ in the range $5-10 \mathrm{~K}$ is observed. It is more instructive to consider the magnetization in field measured at $2 \mathrm{~K}$, shown in Figure 4(b). The samples still show clear ferromagnetic hysteresis and the saturation magnetization decreases gradually with increasing DPA. This is consistent with the suppression of ferromagnetism with decreasing hole concentration. We note that this set of samples all show insulating behavior, consistent with strong disorder. The conductivity can be understood as hopping as shown in Figs. 4(c) and 4(d) by fitting to a $T^{-0.5}$ dependence [73].

Our results provide direct experimental evidence that ferromagnetism can be suppressed with $T_{C}$ below $10 \mathrm{~K}$ by introducing hole compensation in $(\mathrm{Ga}, \mathrm{Mn}) \mathrm{As}$ samples with large Mn concentration. This is easy to understand within the valence-band picture. In sample Mn6ag with $x=0.06$, the Mn-derived impurity band is merged with the valence band before we introduce hole compensation. If we introduce compensation, the Fermi energy shifts up into the tail of the valence band. With decreasing hole concentration, the Fermi energy shifts deeper and deeper into this tail. States at the Fermi energy are thus more and more localized. Ferromagnetism is mainly determined by the properties of the hole states on the length scale of the typical Mn-Mn nearest-neighbor distance, which change smoothly with the localization length and thus with compensation. Since the Mn concentration is high, the Mn-Mn distance is typically smaller than the effective Bohr radius of an acceptor-bound state. In this sense, the Zener $p$ - $d$ exchange can still happen, leading to the formation of Zhang-Rice magnetic polarons $[74,75]$. The temperature below which the magnetic polarons overlap and ferromagnetic order develops depends on the hole concentration. Consequently, $T_{C}$ can be decreased to close to zero Kelvin when the hole concentration is gradually reduced.

\section{SUMMARY AND CONCLUSIONS}

We have presented the magnetic and transport properties of a broad range of (Ga,Mn)As-based DFS samples. The DFS samples used in this study are of state-of-theart quality, as shown by their high $T_{C}$ and confirmed by $\mathrm{X}$-ray diffraction measurements $[51,76]$. We have observed a monotonic decrease of $T_{C}$ with decreasing hole concentration over a large temperature range and the existence of Curie temperatures below $10 \mathrm{~K}$ for heavily compensated samples. These observations cannot be explained if there is a pronounced dip in the DOS between the Mn impurity derived state and the GaAs valence band. Our results rather support the valence-band picture for high-quality (Ga,Mn)As-based DFS. But how can we then understand the experimental results reported in Refs. 25, 37, 38, and 60, which are not consistent with the valence-band picture? We first note that in Refs. 25,37 , and 38 , the total Mn concentrations are $5 \%$, $4.5 \%$, and $6.8 \%$, but the corresponding $T_{C}$ is only around
$60 \mathrm{~K}, 80 \mathrm{~K}$, and $90 \mathrm{~K}$, respectively. This means that the starting materials were already highly compensated by defects. The large influence of a small concentration of $\mathrm{P}$ reported in Ref. 60 was later explained by the presence of additional defects arising with $\mathrm{P}$ co-alloying by ion implantation and pulsed laser annealing [63]. Given the fact that the preparation of ferromagnetic $(\mathrm{Ga}, \mathrm{Mn}) \mathrm{As}$ is a rather delicate procedure, a small deviation in growth parameters can significantly alter the properties of the prepared materials [29]. A reliable conclusion can only be obtained by working with highest-quality $(\mathrm{Ga}, \mathrm{Mn}) \mathrm{As}$ materials [77].

Finally, we return to the question alluded to in the beginning: Is there hope to realize DFS with $T_{C}$ above room temperature? Within the valence-band picture with $p$ $d$ exchange, based on the present results, we suggest to search for materials (a) with smaller lattice constants, which lead to a larger $p$ - $d$ exchange [3] and (b) with a large concentration of mobile holes. The criterion (a) is usually positively correlated with a wide bandgap of the host semiconductor. For $\mathrm{Mn}$ in III-V semiconductors, unfortunately the two criteria cannot be fulfilled simultaneously, since the $p$ - $d$ hybridization increases the binding energy of $\mathrm{Mn}$ acceptors going from arsenides to phosphides and finally nitrides. In samples with strong $p$ $d$ hybridization, the holes are more localized. Moreover, it is generally difficult to achieve p-type doping in widebandgap semiconductors, particularly in $\mathrm{GaN}$ and $\mathrm{ZnO}$ $[78,79]$, even by doping with shallower acceptors than Mn. Searching for different transition-metal dopants is less promising, since other transition-metal ions substituted for the cation tend to have several energy levels of both donor and acceptor type. It has turned out that up to now $(\mathrm{Ga}, \mathrm{Mn})$ As yields the best compromise and has achieved the highest $T_{C}[3,80,81]$. Nevertheless, an intentional introduction of nanoscale inhomogeneities has been proposed as a path to achieve high Curie temperatures in diluted systems [82-84]. Moreover, Mn-doped I-II-V compounds [85] with decoupled spin and charge doping might provide an alternative test bed for producing DFS with high $T_{C}$. At the present stage, $T_{C}$ reaches $220 \mathrm{~K}$ in $\left(\mathrm{Ba}_{0.7} \mathrm{~K}_{0.3}\right)\left(\mathrm{Zn}_{0.85} \mathrm{Mn}_{0.15}\right)_{2} \mathrm{As}_{2}$ [86].

\section{ACKNOWLEDGMENTS}

Financial support by the Helmholtz Association (VHNG-713) is gratefully acknowledged. C.T. acknowledges financial support by the Deutsche Forschungsgemeinschaft.

\section{Appendix A: He ion irradiation}

The samples listed in table I were irradiated with $\mathrm{He}$ ions. The sample notation and experimental condition are listed in table II. 
TABLE II. Sample notations, energy and fluence of He ions.

\begin{tabular}{|c|c|c|c|}
\hline Samples & Energy & Fluence $\left(\mathrm{cm}^{-2}\right)$ & Peak DPA \\
\hline Mn6ann_0 & - & 0 & 0 \\
\hline Mn6ann_1 & $4 \mathrm{keV}$ & $2.0 \times 10^{12}$ & $0.13 \times 10^{-3}$ \\
\hline Mn6ann_2 & $4 \mathrm{keV}$ & $5.0 \times 10^{12}$ & $0.32 \times 10^{-3}$ \\
\hline Mn6ann_3 & $4 \mathrm{keV}$ & $1.0 \times 10^{13}$ & $0.64 \times 10^{-3}$ \\
\hline Mn6ann_4 & $4 \mathrm{keV}$ & $1.5 \times 10^{13}$ & $0.96 \times 10^{-3}$ \\
\hline Mn6ann_5 & $4 \mathrm{keV}$ & $2.0 \times 10^{13}$ & $1.28 \times 10^{-3}$ \\
\hline Mn6ann_6 & $4 \mathrm{keV}$ & $2.5 \times 10^{13}$ & $1.60 \times 10^{-3}$ \\
\hline Mn6ann_7 & $4 \mathrm{keV}$ & $3.0 \times 10^{13}$ & $1.92 \times 10^{-3}$ \\
\hline Mn6ann_8 & $4 \mathrm{keV}$ & $3.5 \times 10^{13}$ & $2.24 \times 10^{-3}$ \\
\hline Mn6ann_9 & $4 \mathrm{keV}$ & $4.0 \times 10^{13}$ & $2.56 \times 10^{-3}$ \\
\hline Mn6ag_0 & - & 0 & 0 \\
\hline Mn6ag_1 & $650 \mathrm{keV}$ & $1.0 \times 10^{14}$ & $0.25 \times 10^{-3}$ \\
\hline Mn6ag_2 & $650 \mathrm{keV}$ & $3.0 \times 10^{14}$ & $0.75 \times 10^{-3}$ \\
\hline Mn6ag_3 & $650 \mathrm{keV}$ & $6.0 \times 10^{14}$ & $1.50 \times 10^{-3}$ \\
\hline Mn6ag_4 & $650 \mathrm{keV}$ & $10.0 \times 10^{14}$ & $2.50 \times 10^{-3}$ \\
\hline Mn6ag_5 & $4 \mathrm{keV}$ & $2.0 \times 10^{13}$ & $1.28 \times 10^{-3}$ \\
\hline Mn6ag_6 & $4 \mathrm{keV}$ & $2.5 \times 10^{13}$ & $1.60 \times 10^{-3}$ \\
\hline Mn6ag_7 & $4 \mathrm{keV}$ & $3.0 \times 10^{13}$ & $1.92 \times 10^{-3}$ \\
\hline Mn6ag_8 & $4 \mathrm{keV}$ & $3.5 \times 10^{13}$ & $2.24 \times 10^{-3}$ \\
\hline Mn6ag_9 & $4 \mathrm{keV}$ & $4.0 \times 10^{13}$ & $2.56 \times 10^{-3}$ \\
\hline Mn10ann_0 & - & 0 & 0 \\
\hline Mn10ann_1 & $4 \mathrm{keV}$ & $5.0 \times 10^{12}$ & $0.32 \times 10^{-3}$ \\
\hline Mn10ann_2 & $4 \mathrm{keV}$ & $1.0 \times 10^{13}$ & $0.64 \times 10^{-3}$ \\
\hline Mn10ann_3 & $4 \mathrm{keV}$ & $1.5 \times 10^{13}$ & $0.96 \times 10^{-3}$ \\
\hline Mn10ann_4 & $4 \mathrm{keV}$ & $2.0 \times 10^{13}$ & $1.28 \times 10^{-3}$ \\
\hline Mn10ann_5 & $4 \mathrm{keV}$ & $2.5 \times 10^{13}$ & $1.60 \times 10^{-3}$ \\
\hline Mn10ann_6 & $4 \mathrm{keV}$ & $3.0 \times 10^{13}$ & $1.92 \times 10^{-3}$ \\
\hline Mn10ann_7 & $4 \mathrm{keV}$ & $3.5 \times 10^{13}$ & $2.24 \times 10^{-3}$ \\
\hline Mn10ann_8 & $4 \mathrm{keV}$ & $4.0 \times 10^{13}$ & $2.56 \times 10^{-3}$ \\
\hline Mn10ann_9 & $4 \mathrm{keV}$ & $4.5 \times 10^{13}$ & $2.88 \times 10^{-3}$ \\
\hline Mn6P6ann_0 & - & 0 & 0 \\
\hline Mn6P6ann_1 & $4 \mathrm{keV}$ & $2.0 \times 10^{12}$ & $0.13 \times 10^{-3}$ \\
\hline Mn6P6ann_2 & $4 \mathrm{keV}$ & $5.0 \times 10^{12}$ & $0.32 \times 10^{-3}$ \\
\hline Mn6P6ann_3 & $4 \mathrm{keV}$ & $1.0 \times 10^{13}$ & $0.64 \times 10^{-3}$ \\
\hline Mn6P6ann_4 & $4 \mathrm{keV}$ & $1.5 \times 10^{13}$ & $0.96 \times 10^{-3}$ \\
\hline Mn6P6ann_5 & $4 \mathrm{keV}$ & $2.0 \times 10^{13}$ & $1.28 \times 10^{-3}$ \\
\hline Mn6P6ann_6 & $4 \mathrm{keV}$ & $2.5 \times 10^{13}$ & $1.60 \times 10^{-3}$ \\
\hline Mn6P6ann_7 & $4 \mathrm{keV}$ & $3.0 \times 10^{13}$ & $1.92 \times 10^{-3}$ \\
\hline Mn6P6ann_8 & $4 \mathrm{keV}$ & $3.5 \times 10^{13}$ & $2.24 \times 10^{-3}$ \\
\hline Mn6P6ann_9 & $4 \mathrm{keV}$ & $4.0 \times 10^{13}$ & $2.56 \times 10^{-3}$ \\
\hline Mn6P9ann_0 & - & 0 & 0 \\
\hline Mn6P9ann_1 & $4 \mathrm{keV}$ & $5.0 \times 10^{12}$ & $0.32 \times 10^{-3}$ \\
\hline Mn6P9ann_2 & $4 \mathrm{keV}$ & $1.0 \times 10^{13}$ & $0.64 \times 10^{-3}$ \\
\hline Mn6P9ann_3 & $4 \mathrm{keV}$ & $2.0 \times 10^{13}$ & $1.28 \times 10^{-3}$ \\
\hline Mn6P9ann_4 & $4 \mathrm{keV}$ & $3.0 \times 10^{13}$ & $2.56 \times 10^{-3}$ \\
\hline
\end{tabular}
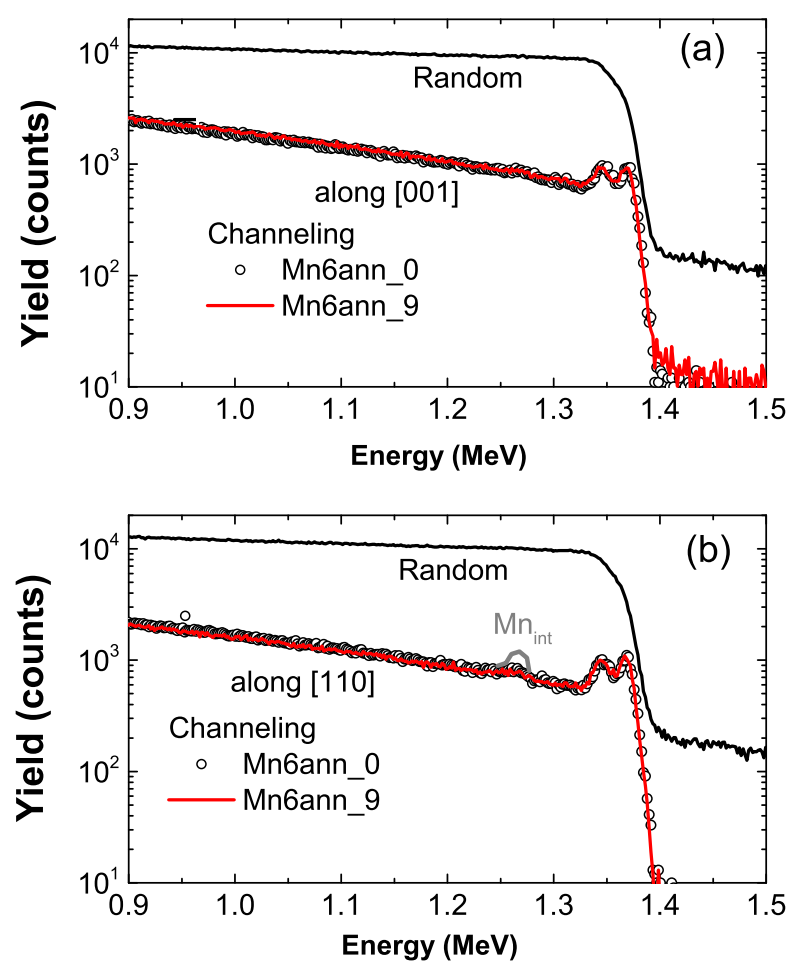

FIG. 5. RBS/Channeling results for sample Mn6ann, comparing virgin (Mn6ann_0) and irradiated (Mn6ann_9) samples. The channeling spectra do not reveal any detectable increase in yield along both [001] (a) and [110] (b). The grey curve schematically drawn in (b) indicates the expected spectrum if there is a lot of $\mathrm{Mn}$ interstitials after irradiation.

\section{Appendix B: Rutherford backscattering spectrometry/channeling}

Rutherford backscattering spectrometry/channeling (RBS) is sensitive to the crystalline disorder and is able to quantitatively measure the fraction of substitutional and interstitial Mn ions [48, 50]. Since the interstitial Mn ions can be invisible along $\mathrm{GaAs}[001]$ [48, 50], we measure the channeling spectra along both GaAs[001] and [110] axis. The RBS measurements were performed with a collimated $1.7 \mathrm{MeV} \mathrm{He}{ }^{+}$beam at a backscattering angle of $170^{\circ}$. The sample was mounted on a three-axis goniometer with a precision of $0.01^{\circ}$. The channeling spectra were measured by aligning the samples to make the impinging $\mathrm{He}^{+}$beam parallel to the GaAs[001] or [110] axis. Figure 5 shows a measurement for samples Mn6ann before and after ion irradiation (up to the largest He ion fluence). Within the detection limit, there is no signature of an increase in the number of interstitial Mn ions. In Fig. 5(b), the expected channeling spectrum [50] in the presence of interstitial Mn ions is schematically drawn on top of the experiment spectrum.

Moreover, the particle-induced X-ray emission (PIXE) 


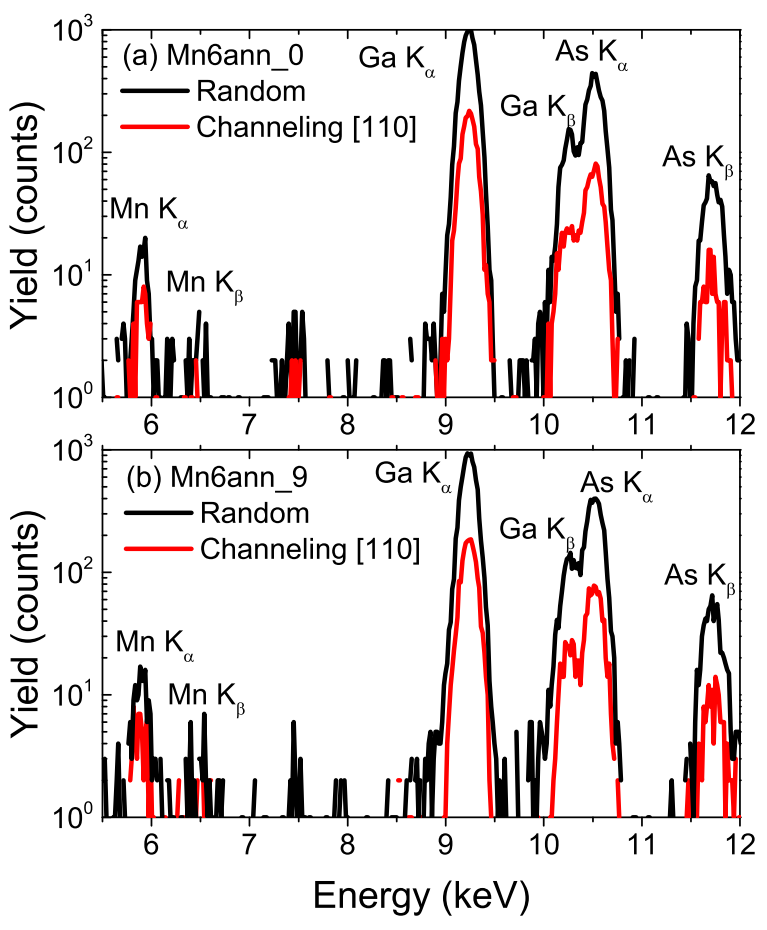

FIG. 6. PIXE/Channeling results for sample Mn6ann along GaAs[110]: (a) Mn6ann_0 before irradiation and (b) Mn6ann_9 after irradiation with largest fluence. In both samples, the channeling effect is clearly revealed.

was recorded simultaneously during RBS measurements. The advantage is that the Mn $K$-lines are well seperated from the Ga and As $K$-lines. However, the emission is relatively weak since we use He ions instead of proton [48]. Figure 6 shows the PIXE/channeling spectra along GaAs[110] for both samples Mn6ann_0 and Mn6ann_9. Although the signal from Mn $K$-lines is weak and noisy, the channeling effect is clearly revealed: the emission is much dropped when the beam is along GaAs[110] (the red spectra in the figure). One can roughly estimate the nonrandom fraction $\left(f_{n r}\right)$ of $\mathrm{Mn}$ using $f_{n r}=\left(1-\chi_{M n} /\left(1-\chi_{G a}\right)\right.$. Here $\chi_{M n}\left(\chi_{G a}\right)$ is the ratio between the integral intensity of the $\mathrm{Mn} K_{\alpha}$ $\left(\mathrm{Ga} K_{\alpha}\right)$ at the channeling geometry and at the random geometry. For both samples, $f_{n r}$ is estimated to be around $75 \%$. This number can be considered as a measure of the substitutional fraction of Mn. However, $f_{n r}$ can be smaller than the Mn substitutional fraction due to the flux peaking effect $[48,50]$. Nevertheless, $f_{n r}$ along GaAs[110] remains the same after He ion irradiation up to the largest fluence.

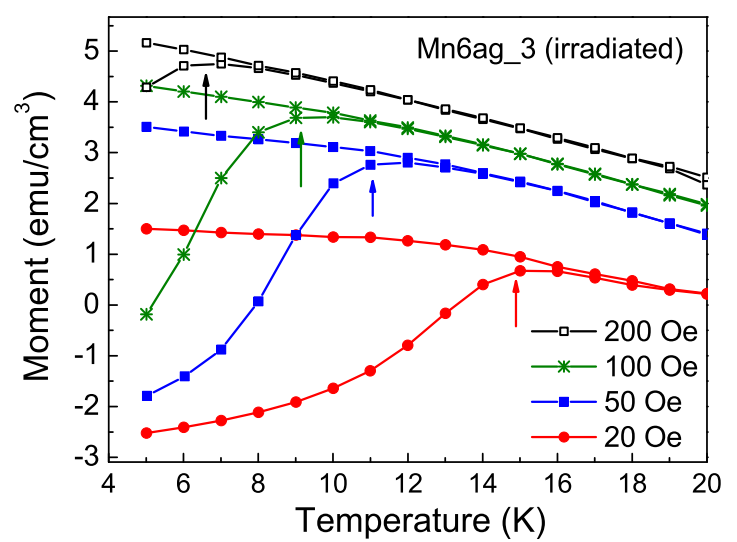

FIG. 7. ZFC/FC magnetization curves for the sample Mn6ag after irradiation. The measurements were performed in different fields. The curves are vertically shifted for better visibility. The disparity between the ZFC and FC curves is the signature of superparamagnetism. The blocking temperatures (indicated by arrows) are shifted to lower temperatures with increasing measurement field.

\section{Appendix C: Superparamagnetism in highly compensated (Ga,Mn)As}

As noted in Sec. IIIC, carrier-mediated ferromagnetism is strongly influenced by the vicinity of the IMT. In particular, spatial fluctuations in the effective Mn and hole concentrations are expected to lead to the formation of paramagnetic or superparamagnetic regions, as the hole concentration is reduced. We have indeed observed signatures for paramagnetic and superparamagnetic components in sample Mn6ag after irradiation.

The appearance of a superparamagnetic component is demonstrated by measuring the zero-field-cooled/fieldcooled (ZFC/FC) temperature-dependent magnetization for sample Mn6ag_3, which is heavily compensated but still shows magnetic hysteresis. In the ZFC measurement, the sample was cooled down from around $300 \mathrm{~K}$ to $5 \mathrm{~K}$ in zero field. Then a small field (in this work 20, 50, 100, 200 Oe, respectively) was applied. The magnetization was measured during warming up to $300 \mathrm{~K}$. Then the field was kept and the sample was cooled from $300 \mathrm{~K}$ to $5 \mathrm{~K}$. During cooling, the FC magnetization was recorded. A similar approach was used in Ref. 23. If there is a superparamagnetic component, one expects to observe a difference between the ZFC and FC curves, as is indeed found in Fig. 7. The maximum in ZFC magnetization occurs at the so-called blocking temperature, which depends on the size of superparamagnetic particles and on the external field. For larger external field, the singledomain superparamagnetic clusters can flip into the field direction at lower temperature. In our measurement, the blocking temperature decreases as the external field is increased from 20 Oe to 200 Oe. 


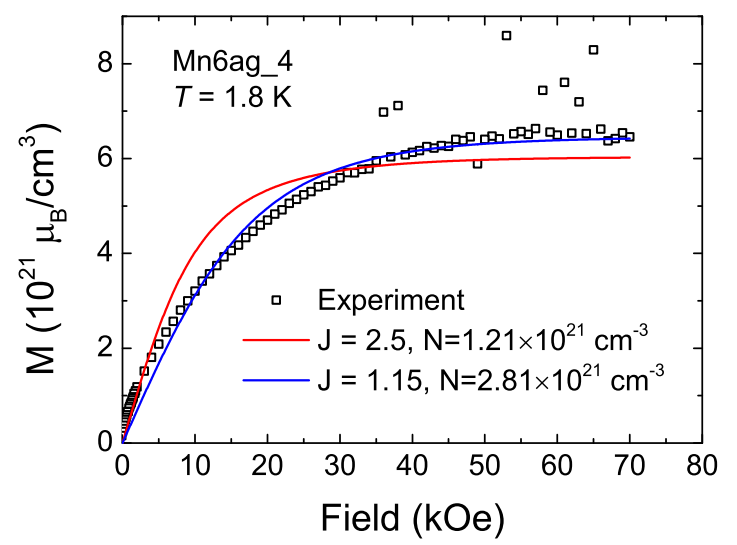

FIG. 8. Magnetization measured at $1.8 \mathrm{~K}$ for sample Mn6ag_4 (the most highly compensated sample). The diamagnetic background has been subtracted. The solid lines are fits using the Brillouin function. The fitting results are also shown in the figure.

Figure 8 shows the magnetization for sample Mn6ag_4 measured at $1.8 \mathrm{~K}$ after subtracting the diamagnetic background. This sample does not show any ferromagnetic hysteresis down to $1.8 \mathrm{~K}$. At $1.8 \mathrm{~K}$, the magnetization does not show saturation up to a field of $7 \mathrm{~T}$. We fit the magnetization by a Brillouin function,

$$
\begin{aligned}
M(\alpha)= & N J \mu_{B} g\left[\frac{2 J+1}{2 J} \operatorname{coth}\left(\frac{2 J+1}{2 J} \alpha\right)\right. \\
& \left.-\frac{1}{2 J} \operatorname{coth}\left(\frac{\alpha}{2 J}\right)\right]
\end{aligned}
$$

where the $g$ factor is about 2 if assuming $\mathrm{Mn}^{2+}\left(d^{5}\right)$ without holes [87, 88], $\mu_{B}$ is the Bohr magneton, $\alpha=$ $g J \mu_{B} H / k_{B} T, k_{B}$ is the Boltzmann constant, and $N$ is the density of spins.

As shown in Fig. 8, the fit for fixed $J=2.5$ does not well reproduce the experimental data. A better fit is obtained for $J=1.15$. This can be explained by considering another component of paramagnetism arising from intrinsic defects in the GaAs substrate [89], which technically cannot be subtracted from the magnetization measurement since the defect concentration can be different depending on the growth condition. This component can be represented by spin $1 / 2(J=S=0.5)$ paramagnetism. Moreover, the other states of Mn impurities could have different $g$ factor and $J$ value [88]. Therefore, it is difficult to accurately separate different paramagnetic components and is beyond the scope of this paper. Nevertheless, a fully compensated (Ga,Mn)As sample shows paramagnetic behavior.
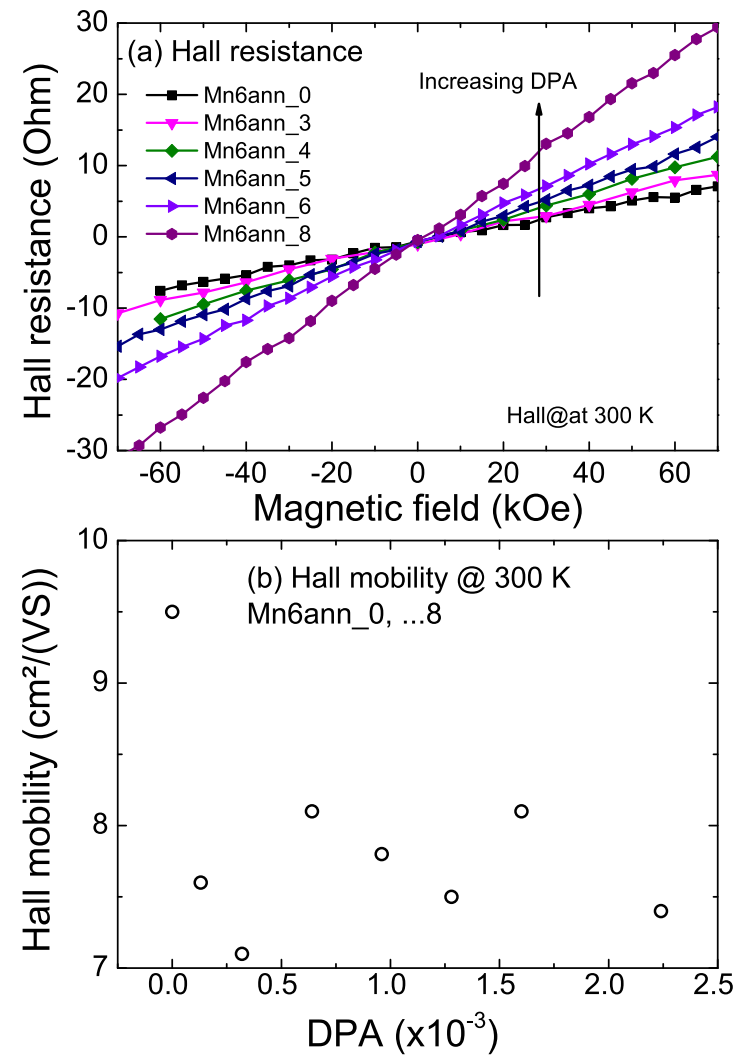

FIG. 9. (a) Hall resistance vs. applied field measured for several samples from Mn6ann after irradiation and (b) Hall mobility for the same set of samples. All samples show ptype conductivity and the hole concentration decreases gradually. The Hall mobility decreases after irradiation but remains rather independent of DPA.

\section{Appendix D: Hole concentration}

Figure 9(a) shows the Hall resistance measured at $300 \mathrm{~K}$ for sample Mn6ann. For increasing DPA, the samples remain p-type conducting, but the Hall resistance increases, indicating a decrease of the hole concentration. Estimated from the Hall measurement shown in Fig. 9(a), the hole concentration gradually decreases from around $2.6 \times 10^{20} \mathrm{~cm}^{-3}$ for the virgin sample to $5.6 \times 10^{19} \mathrm{~cm}^{-3}$ for sample Mn6ann_8. However, due to the paramagnetic component in the Hall resistance [51], the hole concentration is significantly underestimated. We have also estimated the Hall mobility, which decreases after irradiation but remains rather independent of DPA, see Fig. 9(b). However, one has to note the possible overestimation of the Hall mobility due to the underestimated hole concentration.

The initial hole concentration is around $1 \times 10^{21} \mathrm{~cm}^{-3}$, which has been measured by the sample preparation group at low temperature and high field on a similar sample [51]. This value can also be confirmed from our 

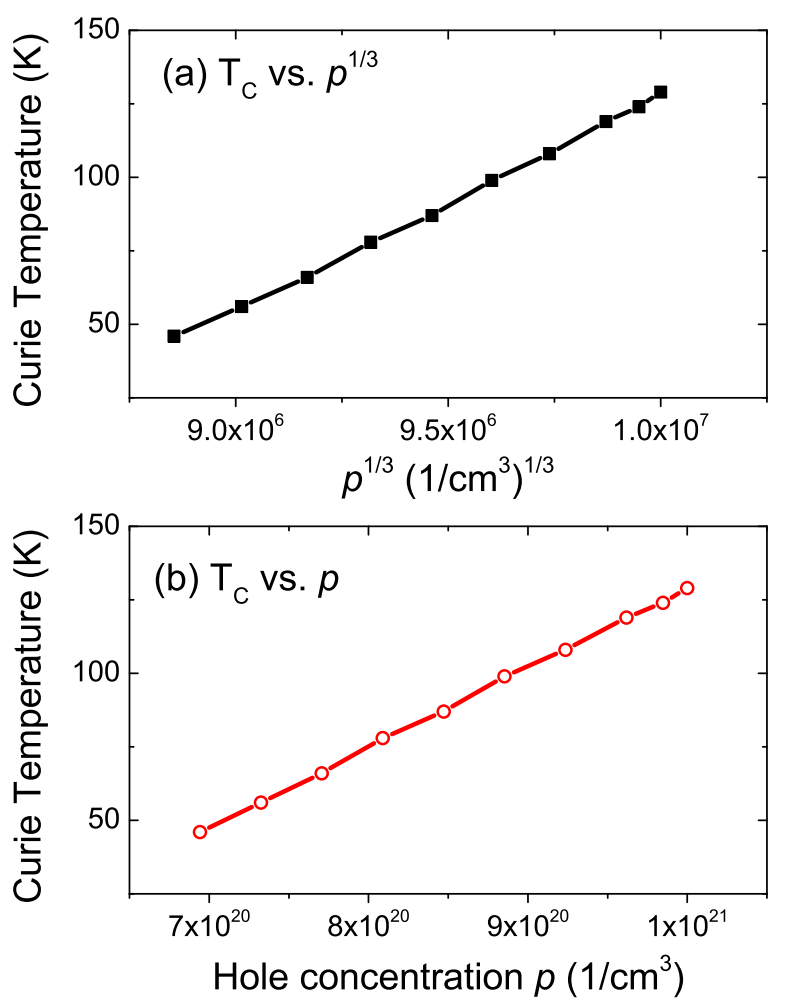

FIG. 10. Curie temperature vs. hole concentration $p$ : (a) $p^{1 / 3}$ and (b) $p$ for samples Mn6ann_0, 9. The hole concentration is estimated by following the approach in Ref. 37 .
PIXE/channeling measurement. If we assume the nonrandom Mn fraction as the substitutional fraction (75\%) and most compensating $\mathrm{Mn}$ interstials have been kicked out during the postgrowth, long-time low-temperature annealing, the hole concentration is $2.21 \times 10^{22} \mathrm{~cm}^{-3} \times$ $0.06 \times 0.75=0.99 \times 10^{21} \mathrm{~cm}^{-3}$. The hole compensation rate by ion irradiation is estimated to be 0.91 hole per vacancy [37]. Then we can calculate the hole concentration $p$ for sample Mn6ann after irradiation and plot $T_{C}$ vs. $p^{1 / 3}$ as shown in Figure 10 . As one can see, $T_{C}$ reveals a linear dependence on $p^{1 / 3}$. However, the dependence is not sensitive to the power $1 / 3$ or 1 when the hole concentration is large.
[1] T. Dietl and H. Ohno, Rev. Mod. Phys. 86, 187 (2014).

[2] T. Jungwirth, J. Sinova, J. Mašek, J. Kučera, and A. H. MacDonald, Rev. Mod. Phys. 78, 809 (2006).

[3] T. Dietl, Nature Materials 9, 965 (2010).

[4] C. Timm, in Handbook of Spin Transport and Magnetism, edited by E. Y. Tsymbal and I. Žutić (CRC Press, 2012) pp. 385-404.

[5] G. Bouzerar and R. Bouzerar, Comptes Rendus Physique 16, 731 (2015).

[6] Y. Ohno, D. K. Young, B. Beschoten, F. Matsukura, H. Ohno, and D. D. Awschalom, Nature 402, 790 (1999).

[7] D. Chiba, M. Yamanouchi, F. Matsukura, and H. Ohno, Science 301, 943 (2003).

[8] M. Ciorga, M. Schlapps, A. Einwanger, S. Geißler, J. Sadowski, W. Wegscheider, and D. Weiss, New J. Phys. 9, 351 (2007).

[9] M. Ciorga, A. Einwanger, U. Wurstbauer, D. Schuh, W. Wegscheider, and D. Weiss, Phys. Rev. B 79, 165321 (2009).

[10] I. Stolichnov, S. W. E. Riester, H. J. Trodahl, N. Setter, A. W. Rushforth, K. W. Edmonds, R. P. Campion, C. T. Foxon, B. L. Gallagher, and T. Jungwirth, Nature Materials 7, 464 (2008).

[11] V. L. Korenev, I. A. Akimov, S. V. Zaitsev, V. F. Sapega, L. Langer, D. R. Yakovlev, Y. A. Danilov, and M. Bayer, Nature Communications 3, 959 (2012).
[12] L. Vila, R. Giraud, L. Thevenard, A. Lemaître, F. Pierre, J. Dufouleur, D. Mailly, B. Barbara, and G. Faini, Phys. Rev. Lett. 98, 027204 (2007).

[13] B. Endres, M. Ciorga, M. Schmid, M. Utz, D. Bougeard, D. Weiss, G. Bayreuther, and C. Back, Nature communications 4 (2013).

[14] J. Gorchon, J. Curiale, A. Lemaître, N. Moisan, M. Cubukcu, G. Malinowski, C. Ulysse, G. Faini, H. von Bardeleben, and V. Jeudy, Phys. Rev. Lett. 112, 026601 (2014).

[15] L. Chen, X. Yang, F. Yang, J. Zhao, J. Misuraca, P. Xiong, and S. von Molnar, Nano Letters 11, 2584 (2011).

[16] A. Zunger, in Solid State Physics, Vol. 39, edited by H. Ehrenreich and D. Turnbull (Academic Press, Orlando, 1986) p. 275.

[17] P. Mahadevan and A. Zunger, Phys. Rev. B 68, 075202 (2003).

[18] K. S. Burch, D. B. Shrekenhamer, E. J. Singley, J. Stephens, B. L. Sheu, R. K. Kawakami, P. Schiffer, N. Samarth, D. D. Awschalom, and D. N. Basov, Phys. Rev. Lett. 97, 087208 (2006).

[19] R. Bouzerar, G. Bouzerar, and T. Ziman, Phys. Rev. B 73, 024411 (2006).

[20] T. Jungwirth, J. Sinova, A. H. MacDonald, B. L. Gallagher, V. Novák, K. W. Edmonds, A. W. Rush- 
forth, R. P. Campion, C. T. Foxon, L. Eaves, E. Olejnik, J. Mašek, S.-R. E. Yang, J. Wunderlich, C. Gould, L. W. Molenkamp, T. Dietl, and H. Ohno, Phys. Rev. B 76, 125206 (2007).

[21] G. Acbas, M.-H. Kim, M. Cukr, V. Novák, M. A. Scarpulla, O. D. Dubon, T. Jungwirth, J. Sinova, and J. Cerne, Phys. Rev. Lett. 103, 137201 (2009).

[22] T. Jungwirth, P. Horodyská, N. Tesařová, P. Němec, J. Šubrt, P. Malý, P. Kužel, C. Kadlec, J. Mašek, I. Němec, M. Orlita, V. Novák, K. Olejnik, Z. Šobáň, P. Vašek, P. Svoboda, and J. Sinova, Phys. Rev. Lett. 105, 227201 (2010).

[23] M. Sawicki, D. Chiba, A. Korbecka, Y. Nishitani, J. A. Majewski, F. Matsukura, T. Dietl, and H. Ohno, Nature Physics 6, 22 (2010).

[24] S. Ohya, K. Takata, and M. Tanaka, Nature Physics 7, 342 (2011).

[25] M. Dobrowolska, K. Tivakornsasithorn, X. Liu, J. K. Furdyna, M. Berciu, K. M. Yu, and W. Walukiewicz, Nature Materials 11, 444 (2012).

[26] N. Samarth, Nature Materials 11, 360 (2012).

[27] S. C. Erwin and A. G. Petukhov, Phys. Rev. Lett. 89, 227201 (2002).

[28] T. Dietl, H. Ohno, F. Matsukura, J. Cibert, and D. Ferrand, Science 287, 1019 (2000).

[29] P. Nemec, V. Novak, N. Tesarova, E. Rozkotova, H. Reichlova, D. Butkovicova, F. Trojanek, K. Olejnik, P. Maly, R. P. Campion, B. L. Gallagher, J. Sinova, and T. Jungwirth, Nature Communications 4, 1422 (2013).

[30] G. Bouzerar, T. Ziman, and J. Kudrnovský, Phys. Rev. B 72, 125207 (2005).

[31] R. C. Myers, B. L. Sheu, A. W. Jackson, A. C. Gossard, P. Schiffer, N. Samarth, and D. D. Awschalom, Phys. Rev. B 74, 155203 (2006).

[32] S. T. B. Goennenwein, T. A. Wassner, H. Huebl, M. S. Brandt, J. B. Philipp, M. Opel, R. Gross, A. Koeder, W. Schoch, and A. Waag, Phys. Rev. Lett. 92, 227202 (2004).

[33] L. Thevenard, L. Largeau, O. Mauguin, A. Lemaître, and B. Theys, Appl. Phys. Lett. 87, 182506 (2005).

[34] K. Khazen, H. von Bardeleben, J. Cantin, L. Thevenard, L. Largeau, O. Mauguin, and A. Lemaître, Phys. Rev. B 77, 165204 (2008).

[35] L. Thevenard, L. Largeau, O. Mauguin, A. Lemaître, K. Khazen, and H. J. von Bardeleben, Phys. Rev. B 75, 195218 (2007).

[36] H. Ohno, D. Chiba, F. Matsukura, T. Omiya, E. Abe, T. Dietl, Y. Ohno, and K. Ohtani, Nature 408, 944 (2000).

[37] M. A. Mayer, P. R. Stone, N. Miller, H. M. Smith, O. D. Dubon, E. E. Haller, K. M. Yu, W. Walukiewicz, X. Liu, and J. K. Furdyna, Phys. Rev. B 81, 045205 (2010).

[38] E. H. C. P. Sinnecker, G. M. Penello, T. G. Rappoport, M. M. Sant'Anna, D. E. R. Souza, M. P. Pires, J. K. Furdyna, and X. Liu, Phys. Rev. B 81, 245203 (2010).

[39] L. Li, S. D. Yao, S. Zhou, D. Bürger, O. Roshchupkina, S. Akhmadaliev, A. W. Rushforth, R. P. Campion, J. Fassbender, M. Helm, B. L. Gallagher, C. Timm, and H. Schmidt, J. Phys. D: Appl. Phys. 44, 045001 (2011).

[40] S. Pearton, Mater. Sci. Eng. R-Rep. 4, 313 (1990).

[41] P. N. K. Deenapanray, Q. Gao, and C. Jagadish, J. Appl. Phys. 93, 9123 (2003).

[42] T. E. Winkler, P. R. Stone, T. Li, K. M. Yu, A. Bonanni, and O. D. Dubon, Appl. Phys. Lett. 98, 012103 (2011).
[43] B. L. Sheu, R. C. Myers, J.-M. Tang, N. Samarth, D. D. Awschalom, P. Schiffer, and M. E. Flatté, Phys. Rev. Lett. 99, 227205 (2007).

[44] M. Wang, R. P. Campion, A. W. Rushforth, K. W. Edmonds, C. T. Foxon, and B. L. Gallagher, Appl. Phys. Lett. 93, 132103 (2008).

[45] R. Campion, K. Edmonds, L. Zhao, K. Wang, C. Foxon, B. Gallagher, and C. Staddon, J. Cryst. Growth 247, 42 (2003).

[46] L. Chen, S. Yan, P. F. Xu, J. Lu, W. Z. Wang, J. J. Deng, X. Qian, Y. Ji, and J. H. Zhao, Appl. Phys. Lett. 95, 182505 (2009).

[47] J. Ziegler, J. Biersack, and U. Littmark, The Stopping and Range of Ions in Solids (Pergamon, 1985).

[48] K. M. Yu, W. Walukiewicz, T. Wojtowicz, I. Kuryliszyn, X. Liu, Y. Sasaki, and J. K. Furdyna, Phys. Rev. B 65, 201303 (2002).

[49] L. Pereira, U. Wahl, S. Decoster, J. Correia, L. Amorim, M. da Silva, J. Araújo, and A. Vantomme, Phys. Rev. B 86, 125206 (2012).

[50] D. Benzeggouta, K. Khazen, I. Vickridge, H. J. von Bardeleben, L. Chen, X. Z. Yu, and J. H. Zhao, Phys. Rev. B 89, 115323 (2014).

[51] M. Wang, Studies of III-V ferromagnetic semiconductors, Ph.D. thesis, University of Nottingham (2012).

[52] T. Jungwirth, J. Mašek, K. Y. Wang, K. W. Edmonds, M. Sawicki, M. Polini, J. Sinova, A. H. MacDonald, R. P. Campion, L. X. Zhao, N. R. S. Farley, T. K. Johal, G. van der Laan, C. T. Foxon, and B. L. Gallagher, Phys. Rev. B 73, 165205 (2006).

[53] T. Jungwirth, K. Y. Wang, J. Mašek, K. W. Edmonds, J. König, J. Sinova, M. Polini, N. A. Goncharuk, A. H. MacDonald, M. Sawicki, A. W. Rushforth, R. P. Campion, L. X. Zhao, C. T. Foxon, and B. L. Gallagher, Phys. Rev. B 72, 165204 (2005).

[54] A. Lemaître, A. Miard, L. Travers, O. Mauguin, L. Largeau, C. Gourdon, V. Jeudy, M. Tran, and J.M. George, Appl. Phys. Lett. 93, 021123 (2008).

[55] M. Cubukcu, H. von Bardeleben, K. Khazen, J. Cantin, O. Mauguin, L. Largeau, and A. Lemaître, Phys. Rev. B 81, 041202 (2010).

[56] M. Cubukcu, H. J. von Bardeleben, J. L. Cantin, and A. Lemaître, Appl. Phys. Lett. 96, 102502 (2010).

[57] M. Yahyaoui, K. Boujdaria, M. Cubukcu, C. Testelin, and C. Gourdon, J. Phys.: Conden. Matter 25 (2013).

[58] M. Cubukcu, H. von Bardeleben, J. Cantin, I. Vickridge, and A. Lemaitre, Thin Solid Films 519, 8212 (2011).

[59] S. Shihab, H. Riahi, L. Thevenard, H. J. von Bardeleben, A. Lemaître, and C. Gourdon, Appl. Phys. Lett. 106, 142408 (2015).

[60] P. R. Stone, K. Alberi, S. K. Z. Tardif, J. W. Beeman, K. M. Yu, W. Walukiewicz, and O. D. Dubon, Phys. Rev. Lett. 101, 087203 (2008).

[61] M. A. Scarpulla, B. L. Cardozo, R. Farshchi, W. M. H. Oo, M. D. McCluskey, K. M. Yu, and O. D. Dubon, Phys. Rev. Lett. 95, 207204 (2005).

[62] M. Khalid, E. Weschke, W. Skorupa, M. Helm, and S. Zhou, Phys. Rev. B 89, 121301 (2014).

[63] R. Bouzerar, F. Máca, J. Kudrnovský, and L. Bergqvist, Phys. Rev. B 82, 035207 (2010).

[64] G. Bouzerar, T. Ziman, and J. Kudrnovský, EPL (Europhysics Letters) 69, 812 (2005).

[65] A. W. Rushforth, M. Wang, N. R. S. Farley, R. P. Campion, K. W. Edmonds, C. R. Staddon, C. T. Foxon, and 
B. L. Gallagher, J. Appl. Phys. 104, 073908 (2008).

[66] T. Dietl, J. Phys. Soc. Jpn. 77, 031005 (2008).

[67] J. Kudrnovský, I. Turek, V. Drchal, F. Máca, P. Weinberger, and P. Bruno, Phys. Rev. B 69, 115208 (2004).

[68] M. Abolfath, T. Jungwirth, J. Brum, and A. H. MacDonald, Phys. Rev. B 63, 054418 (2001).

[69] D. Ferrand, J. Cibert, A. Wasiela, C. Bourgognon, S. Tatarenko, G. Fishman, T. Andrearczyk, J. Jaroszyński, S. Koleśnik, T. Dietl, B. Barbara, and D. Dufeu, Phys. Rev. B 63, 085201 (2001).

[70] T. Wojtowicz, G. Cywiński, W. L. Lim, X. Liu, M. Dobrowolska, J. K. Furdyna, K. M. Yu, W. Walukiewicz, G. B. Kim, M. Cheon, X. Chen, S. M. Wang, and H. Luo, Appl. Phys. Lett. 82, 4310 (2003).

[71] P. P. Edwards and M. J. Sienko, Phys. Rev. B 17, 2575 (1978).

[72] C. Timm, J. Phys.: Condensed Matter 15, R1865 (2003).

[73] A. Efros and B. Shklovskii, Springer Series in Solid-State Sciences, Springer, Berlin (1984).

[74] T. Dietl, F. Matsukura, and H. Ohno, Phys. Rev. B 66, 033203 (2002).

[75] A. Kaminski and S. Das Sarma, Phys. Rev. Lett. 88, 247202 (2002).

[76] M. Wang, K. W. Edmonds, B. L. Gallagher, A. W. Rushforth, O. Makarovsky, A. Patanè, R. P. Campion, C. T. Foxon, V. Novak, and T. Jungwirth, Phys. Rev. B 87, 121301 (2013).

[77] K. Edmonds, B. Gallagher, M. Wang, A. Rushforth, O. Makarovsky, A. Patane, R. Campion,
C. Foxon, V. Novak, and T. Jungwirth, arXiv preprint arXiv:1211.3860 (2012).

[78] S.-H. Wei, Comput. Mater. Sci. 30, 337 (2004).

[79] A. Zunger, Appl. Phys. Lett. 83 (2003).

[80] R. Bouzerar and G. Bouzerar, EPL (Europhysics Letters) 92, 47006 (2010).

[81] H. Peng, J. Li, and S.-H. Wei, Appl. Phys. Lett. 102, 122409 (2013).

[82] S. Kuroda, N. Nishizawa, K. Takita, M. Mitome, Y. Bando, K. Osuch, and T. Dietl, Nature materials 6, 440 (2007).

[83] G. Bouzerar, T. Ziman, and J. Kudrnovský, Appl. Phys. Lett. 85, 4941 (2004).

[84] A. Chakraborty, R. Bouzerar, S. Kettemann, and G. Bouzerar, Phys. Rev. B 85, 014201 (2012).

[85] Z. Deng, C. Jin, Q. Liu, X. Wang, J. Zhu, S. Feng, L. Chen, R. Yu, C. Arguello, T. Goko, et al., Nature communications 2, 422 (2011).

[86] K. Zhao, B. Chen, G. Zhao, Z. Yuan, Q. Liu, Z. Deng, J. Zhu, and C. Jin, Chin. Sci. Bull. 59, 2524 (2014).

[87] J. Schneider, U. Kaufmann, W. Wilkening, M. Baeumler, and F. Köhl, Phys. Rev. Lett. 59, 240 (1987).

[88] M. Pawlowski, M. Piersa, A. Wolos, M. Palczewska, G. Strzelecka, A. Hruban, J. Gosk, M. Kaminska, and A. Twardowski, Acta Physica Polonica A 108, 825 (2006).

[89] B. K. Meyer, J. M. Spaeth, and M. Scheffler, Phys. Rev. Lett. 52, 851 (1984). 